\title{
ARTICLES
}

\section{EPILEPSY MANAGEMENT AT HOME BY CAREGIVERS}

Ms. Marie Elezabeth Pinto* \& Dr. Ian Clement**

*Ph.D. Scholar, Himalayan University, Itanagar, Arunachal Pradesh, India.

**Professor cum Vice-principal, Bhai Gurdas Institute of Nursing, Sangrur, Punjab, India. DOI: http://doi.org/10.47211/trr.2019.v05i02.013

Received $15^{\text {th }}$ November 2019, Accepted $25^{\text {th }}$ November 2019, Published $20^{\text {th }}$ December 2019

\section{ABSTRACT:}

Epilepsy is a brain disorder characterized by recurrent seizures which arise for cerebral, presenting with episodes of sensory, motor or autonomic rounds with or without loss of consciousness. Main problem is the unpredictability of when and where the next seizure will occur. Sadly, it is also one of the most misunderstood conditions. People may have strange sensations and emotions or behave strangely. Some person may have violent muscle spasms or lose consciousness. Continued educational efforts will play a key role in helping people learn to live with and get knowledge of epilepsy. Thus, individuals and families need education and skills building throughout the course of the disorder, particularly during times of change, such as an increased frequency of seizures, changes in treatment.

Key Words: Epilepsy, recurrent seizures, loss of consciousness, management at home.

\section{About the Authors:}

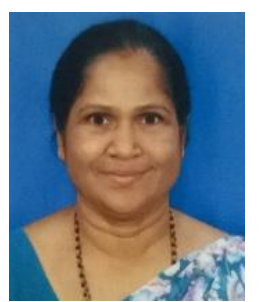

Author Ms. Marie Elezabeth Pinto is research scholar at Himalayan University, Itanagar, Arunachal Pradesh, India.

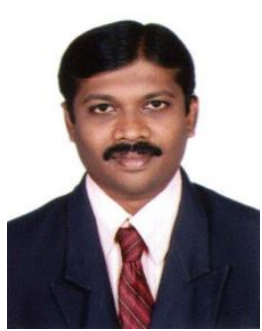

Author Dr. Ian Clement is Professor cum Vice-principal of Bhai Gurdas Institute of Nursing at Sangrur in Punjab, India. He has presented papers in various conferences and seminars. He has many research publications to his Name. 


\section{INTRODUCTION}

Epilepsy is not a disease; it is a sign or symptom of an underlying neurological disorder. The word epilepsy is derived from Greek word epilepsia meaning nothing more than a tendency to have seizures (a condition of being overcome seized or attacked). Epilepsy is a brain disorder characterized by recurrent seizures of cerebral origin, presenting with episodes of sensory, motor or autonomic phenomenon with or without loss of consciousness. Its most disabling aspect is unpredictability of when and where the next seizure will occur. Unfortunately, it is also one of the most misunderstood conditions. People may have strange sensations and emotions or behave strangely. They may have violent muscle spasms or lose consciousness. It is a sudden burst of electricity in your brain, very much like a tiny bolt of lightning. This electricity is a signal that causes one's body to feel different, or makes one's body do things that one can't control. Sometimes the electrical signal only reaches part of one's brain. Then a part of the body, like an arm or a leg, may move on its own. If the signal goes all through the brain, one may shake all over or may fall asleep for a short time. The whole seizure is usually very short - only a few seconds or perhaps a minute or so.

The diagnosis of epilepsy, although given to an individual, affects the entire family and its constellation of friendships and other relationships. At onset all are confronted with the immediate need to learn about the disorder and its management. More of the population is residing in rural areas where the people are not aware of the disease condition. Behavioural management enables the epileptic to live with his/her seizures, and to overcome the psychosocial impacts of the disease. For many people with epilepsy, the myths are about as hard to live with as the medical disorder itself. Even harder is the discrimination, especially in schooling and later in employment that often limits and isolates people with epilepsy. Lack of self-confidence and selfesteem often go along with the disorder as well. Epilepsy is not related to mental illness. A person having a seizure does not need to be restrained. These are all myths that people with epilepsy hear every day. These myths result from both prejudice about and ignorance of epilepsy.

Epilepsy is a disorder with many possible causes. Anything that disturbs the normal pattern of neuron activity - from illness to brain damage to abnormal brain development -- can lead to seizures. Epilepsy may develop because of an abnormality in brain wiring, an imbalance of nerve signalling chemicals called neurotransmitters, or some combination of these factors. Researchers believe that some people with epilepsy have an abnormally high level of excitatory neurotransmitters that increase neuronal activity, while others have an abnormally low level of inhibitory neurotransmitters that decrease neuronal activity in the brain. Epilepsy is the second most common chronic neurological condition seen by neurologists. Its prevalence varies in relation to ethnicity, geography, age and sex. Living with epilepsy, its unpredictable seizures, and its comorbid conditions presents many challenges over time. Educational efforts can play a key role in helping people learn to live with and understand epilepsy and its effects over the life span. Thus, individuals and families need education and skills building throughout the course of the disorder, particularly during times of change, such as an increased frequency of seizures, changes in treatment (e.g., switching medications, starting a new treatment option, discontinuing a medication or other treatment option), and major life transitions (e.g., from youth to adulthood, from adulthood to older adulthood).

Many people especially in rural areas believe that epilepsy is an extraordinary phenomenon; only God or as in other cultures demons or evil spirits could hit someone down. It is a heavily stigmatized disorder, both in the general community and among the medical profession; epilepsy is surrounded by a number of common misconceptions which contribute to poor psychosocial adjustment and problems in the medical management and home care of this condition.

\section{Common causes of epilepsy include:}

- Stroke

- Dementia, such as Alzheimer's disease

- Traumatic brain injury

- Infections, brain abscess, meningitis, encephalitis,

- $\quad$ AIDS

- Brain injury that occurs during or near birth

- Phenylketonuria

- Brain tumour

- Abnormal blood vessels in the brain

- Certain illness that damage or destroy brain tissue 
Epilepsy usually begins between ages 5 and 20, but they can happen at any age. There may be a family history of seizures or epilepsy. This disease can interfere with the physical, emotional and social functioning of people of all ages, particularly if seizures are not controlled.

Seizures may result from electrical activity in any part of the brain and can therefore present in many different ways. They are broadly classified as either generalized or partial seizures.

\section{Antiepileptic Drugs (AED) and Management of Epilepsy}

People with developmental disabilities are generally at greater risk of AED adverse reactions. The decision to treat needs to be based on a careful analysis of the likely impact of seizures on a person's health and quality of life and the likelihood and impact of adverse effects of AED. Factors which complicate treatment with AED:

- Presence of multiple or complex types of seizures

- Increased susceptibility to adverse effects.

- Problems with communicating symptoms of adverse effects

- Intolerance to blood tests

- $\quad$ Limited acceptability of particular AED formulations.

Most people with developmental disability can have their epilepsy controlled by using one or, at most, 2 AEDs. If combination therapy is required, a second AED should be chosen which complements the first drug and attention should be paid to each one's influence on the other's metabolism.

\section{Drugs used in Epilepsy}

- Sodium Valproate is the drug of choice for generalised seizures.

- Carbamazepine is the drug of choice for partial seizures.

- Lamotrigine is increasingly popular and a useful alternative drug for both seizure types

Seizure control is more in the hands of the patient and the caregiver. The prognosis of the epilepsy depends on the understanding and compliance of the patient and person entrusted with the treatment and management of the disease. The parents respond best to the information that focuses on their specific area of concern. Hence for the therapeutic regimen to be beneficial, patient and their care giver must be informed about the disease and motivated to share responsibility in maintaining good health.

\section{Factors Influencing Self-management Care}

- Confidence in one's abilities

- Satisfaction with care

- Communication with doctors and health care providers

- Support

- Emotional well-being or mood

- Stigma

- Feelings of control

- Individualized goals and treatment

Society's misconceptions have a major impact on peoples view towards epilepsy and its management in rural areas in various parts of the country. Parental fear of convulsion is the major problem with serious negative consequences in their daily life. In early times people believed epilepsy as a divine origin and were called the sacred disease because someone with epilepsy was thought to be "seized". Majority of mothers have false belief about epilepsy and they have different knowledge, attitude and practices especially in low socioeconomic families.

As epilepsy is the disorder that is chronic in nature and it requires continuous medical compliance. The nature of the disease involves fits occurring at any time and place, so there are the chances to get injury and social embarrassment which compels the families to adopt certain practices and precautions regarding medication, fit management, education, employment, marriage \& pregnancy of epileptics. These practices are based on the knowledge and attitude of people towards epilepsy. These practices can lead to any accident if not managed properly. To avoid the chances of accidents there should be adequate knowledge regarding epilepsy, its causes, complications, dietary management, medication compliances and skill in first aid management. The investigator is interested to develop skill based knowledge in the family members of epileptic patients regarding home management of epilepsy to avoid any malpractices. So keeping in mind the above facts, study on home management was planned to make the people aware regarding epilepsy, its complications, medical 
compliance, dietary patterns and management of epileptic fit at home. Management of the epileptic patient by family members at home during epileptic fit and other aspects of epilepsy management are needed.

\section{CONCLUSION:}

Accurate information for individuals with epilepsy and their families, education about the consequences of epilepsy, and skills development in self-management are essential components for helping individuals become better partners in patient-cantered care. People with epilepsy and their families are often not educated about epilepsy's risks, including injury and mortality. Parents of children with epilepsy need information about the disorder and available support resources. Children and youth with epilepsy, as they get older, need increasing information about the disorder and its implications for their future. Adults' information priorities relate to employment, driving, and management of stress; and women need information about hormonal influences on the disorder and the potential effects of seizure medications on pregnancy. Older adults also may have specific information needs, given the likelihood they are taking medications for other chronic health conditions and have an increased risk of falls.

\section{REFERENCES}

1. Radhakrishnan, K. and Rao, M.B., Epilepsy in India. Indian Epilepsy Association Mumbai. 2000. pp. 343-62.

2. G Gopinath. (2012), A randomized study on Epilepsy in India. Advanced Nursing Practice. Vol. 2(14).

3. H Guptha. (2013). Epileptic awareness in India, Nursing Journal. (5) 3-4.

4. Henry, Tanya DK, Mishra A, Mathur NB, Badhan S (2010) Seizure disorder care $\neg$ and practice. Mexico, J Med Sci; 60(12). 